\title{
AMENDMENTS
}

\section{Author Correction: Molecular engineering of organic-inorganic hybrid perovskites quantum wells}

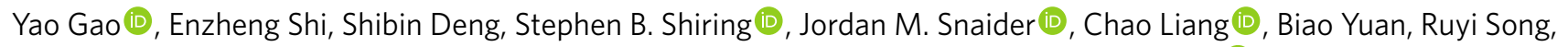
Svenja M. Janke, Alexander Liebman-Peláez, Pilsun Yoo, Matthias Zeller, Bryan W. Boudouris (D), Peilin Liao, Chenhui Zhu, Volker Blum (1), Yi Yu, Brett M. Savoie (D), Libai Huang (1) and Letian Dou (D)

Correction to: Nature Chemistry https://doi.org/10.1038/s41557-019-0354-2, published online 11 November 2019.

In the version of this Article originally published, in the sentence beginning 'For $(4 \mathrm{Tm})_{2} \mathrm{PbI}_{4}$ and $\left(\mathrm{BTm}_{2} \mathrm{PbI}_{4}\right.$, the text '4TCNm' should have read 'BTm'. This has been corrected in the online versions of this Article. In the original crystallographic data for compound $(\mathrm{BTm})_{2} \mathrm{PbI}_{4}$ (CCDC number 1846392) the nitrogen atom on the benzothiadiazole unit was mislabelled as a carbon atom. The CIF has now been updated. This does not influence the conclusions of this paper. The originally published version of the Supplementary Information has been updated to mention the B-level alert for the (BTm) ${ }_{2} \mathrm{PbI}_{4}$ CIF on pages 45 and 48 .

Published online: 20 July 2020

https://doi.org/10.1038/s41557-020-0521-5

(c) The Author(s), under exclusive licence to Springer Nature Limited 2020

\section{Author Correction: Improving reaction prediction}

Ella M. Gale and Derek J. Durand

Correction to: Nature Chemistry https://doi.org/10.1038/s41557-020-0478-4, published online 14 May 2020.

In the version of this Meeting Report originally published, conference co-hosts were missing from the end of the first paragraph. The end of the final paragraph has also been updated to include links to a relevant online website and the newly added reference 13 . This has been corrected in the online versions of this Article.

Published online: 21 August 2020

https://doi.org/10.1038/s41557-020-0545-X

(c) The Author(s), under exclusive licence to Springer Nature Limited 2020

\section{Publisher Correction: Time-dependent enantiodivergent synthesis via sequential kinetic resolution}

Hang-Fei Tu, Pusu Yang, Zi-Hua Lin, Chao Zheng (D) and Shu-Li You (D)

Correction to: Nature Chemistry https://doi.org/10.1038/s41557-020-0489-1, published online 29 June 2020.

In the version of this Article originally published, in Table 1, the methyl group in compound 3af appeared in the wrong position. This incorrectly showed compound 3 af to have the same structure as compound 3ag. This has been corrected in the online versions of this Article.

Published online: 11 August 2020

https://doi.org/10.1038/s41557-020-0546-9

(c) The Author(s), under exclusive licence to Springer Nature Limited 2020 